\title{
Editorial
}

Bundesgesundheitsbl 2016 · 59:1-3

DOI 10.1007/s00103-015-2274-7

Online publiziert: 2. Dezember 2015

c) Springer-Verlag Berlin Heidelberg 2015

CrossMark

Gewalt ist kein Randphänomen. Die Erfahrung von Gewalt durchdringt weltweit ausnahmslos alle Lebensbereiche. Die Weltgesundheitsorganisation (WHO) formuliert dies deutlich im Weltbericht Gewalt und Gesundheit: „Gewalt ist eine Weltgeißel, die das Gefüge von Gemeinschaften zerreißt und Leben, Gesundheit und Glück von uns allen bedroht." Jährlich verlieren 1,6 Mio. Menschen weltweit ihr Leben durch Gewalt. Bezogen auf jede Person, die durch Gewalt stirbt, wird ein Vielfaches an Menschen verletzt, die unter körperlichen, sexuellen und geistigseelischen Beschädigungen, Deprivation oder Vernachlässigung leiden. Prävalenzraten für die deutsche Bevölkerung basieren häufig auf retrospektiven Selbstberichten mit hohem Dunkelfeld und großer Schwankungsbreite. Es berichten jedoch 3 bis $5 \%$ der Erwachsenen von mehrfachen Erfahrungen körperlicher Gewalt und 15 bis $19 \%$ von psychischer Gewalt innerhalb der letzten zwölf Monate oder von starken gesundheitlichen Beeinträchtigungen durch eigene Gewalterfahrung in der Kindheit. Das Risiko für Gewalterfahrungen hängt dabei von verschiedenen Faktoren wie beispielsweise Alter, Geschlecht, psychosozialem Umfeld und dem kulturellen Hintergrund ab.

Gewalt ist nicht auf spezifische Zielgruppen beschränkt. Gewalt und Gesundheit stellt ein komplexes, multidisziplinäres Phänomen dar, und es umfasst auch deutschlandweit alle Kontexte: Familie, Schule, Beruf, Einrichtungen der Altenpflege und das Gesundheitswesen. Frauen wie Männer, Kinder wie Alte sind betroffen als Opfer sowie auch als Täter. Gewalthandlungen finden ebenso in institutionellen Bereichen wie Arbeitsplatz, Psychiatrie, Alteneinrichtungen statt und auch im familiären Umfeld als

\author{
Uwe Koch-Gromus ${ }^{1}$ Silke Pawils ${ }^{2}$ \\ ${ }^{1}$ Dekan der Medizinischen Fakultät, Universitätsklinikum Hamburg-Eppendorf, Hamburg, Deutschland \\ 2 Zentrum für Psychosoziale Medizin, Institut und Poliklinik für Medizinische Psychologie, \\ Universitätsklinikum Hamburg-Eppendorf, Hamburg, Deutschland
}

\section{Gewalt und Gesundheit}

Kindeswohlgefährdung und/oder häusliche Gewalt.

\section{Gewaltformen sind vielfältig und} oft multipel. Die Weltgesundheitsorganisation (WHO) definiert Gewalt als „absichtliche(n) Gebrauch von angedrohtem oder tatsächlichem körperlichem Zwang oder physischer Macht gegen die eigene oder eine andere Person, gegen eine Gruppe oder Gemeinschaft, der entweder konkret oder mit hoher Wahrscheinlichkeit zu Verletzungen, Tod, psychischen Schäden, Fehlentwicklung oder Deprivation führt“. In der Typologie der Gewalt der WHO wird die jeweilige Form der Gewaltausübung spezieller gefasst als: körperliche, psychische, sexuelle Gewalt, Vernachlässigung. Diese einzelnen Gewaltformen kommen jedoch selten isoliert vor, sondern liegen häufig als komplexe Mischformen vor, die sich gegenseitig überlappen und damit ihre gesundheitsschädliche Wirkung verstärken.

Gewalt bleibt nicht ohne gesundheitliche Folgen. Gewalterfahrungen können mit erheblichen psychosozialen Belastungen und gesundheitlichen Konsequenzen verbunden sein, wie körperlichen und seelischen Verletzungen, Rückzug und Isolation, Depression, Angststörungen, sozialer Beeinträchtigungen oder Substanzgebrauch. Mit dem Erleben multipler Gewaltarten ist ein erhöhtes Risiko für einen schlechteren Gesundheitszustand verbunden, besonders wenn Gewalt im Kindesalter erlebt wurde.

Psychosoziale Beratung für Gewaltopfer in der medizinischen Versorgung. Speziell für Gewaltopfer ist es in der medizinischen Versorgung daher wichtig, frühe psycho- soziale Beratung zu erhalten, um eine Reduzierung der psychischen Belastung zu erreichen. Der Zusammenhang zwischen dem eigenen Gesundheitszustand und den erlebten Gewalterfahrungen wird oft von den Gewaltopfern nicht gesehen, und frühzeitige Unterstützung kann der Entwicklung psychopathologischer Auffälligkeiten entgegenwirken oder deren Schwere mindern.

\section{Gewaltprävention ist nötig - und mög-}

lich!. Gewalt muss umfassend und ganzheitlich - mit einem besonderen Augenmerk auf Public Health - verstanden, diskutiert und behandelt werden. Mit dem Bezug auf Public Health wird Gewalt als ein Sozialphänomen verstanden, das letztendlich durch Prävention verhindert werden kann und muss. Ergebnisse der aktuellen Forschung zeigen, dass in Kindheit und Jugend erfahrene Gewalt mit einem erhöhten Risiko verbunden ist, im späteren Lebensalter erneut Opfer von Gewalt zu werden, aber auch selbst Gewalt auszuüben. Für Gewaltprävention im Kindesalter - etwa im Rahmen Früher Hilfen - und insbesondere für Schulprogramme konnte vielfach die Wirksamkeit belegt werden. Eine flächendeckende Umsetzung in Kommunen und ganzen Bundesländern setzt sich diesbezüglich immer mehr durch. Beratungsangebote für erwachsene Gewaltopfer in Hausarztpraxen, an Kliniken und in Alteneinrichtungen können wirksam als Schutzfaktor für Gewaltfolgen im Sinne psychischer Erkrankungen dienen und finden langsam Verbreitung.

Insgesamt ist Gewaltprävention für alle gesellschaftlichen Bereiche gleichsam mit der Verbesserung der Behandlung und Beratung von Gewaltopfern und -tätern in Forschung und Praxis zu diskutieren. 
Im hier vorliegenden Heft des Bundesgesundheitsblatts wird der Themenschwerpunkt "Gesundheit und Gewalt" unter allen genannten unterschiedlichen Perspektiven und bei verschiedenen Gruppen von Betroffenen zur Diskussion gestellt.

Im ersten Themenblock werden in 4 Beiträgen Prävalenzen, Gewaltformen und Folgen von Gewalt übergreifend behandelt. Cornelia Lange, Anne Starker, Elena von der Lippe und Heike Hölling stellen die auf der Grundlage der bevölkerungsrepräsentativen Studie zur Gesundheit Erwachsener in Deutschland (DEGS1) gefundenen Ergebnisse zur psychischen und körperlichen Gewalt dar. Ihre Analysen zeigen, dass sowohl Opfer wie Täter körperlicher oder psychischer Gewalt ein geringeres psychisches Wohlbefinden aufweisen. Erfahrungen von Gewalt in der Kindheit und Jugend erhöhen das Risiko, später Gewaltopfer oder Gewalttäter zu werden.

Ute Habel et al. zielen mit ihrer Prävalenzstudie aufSymptome und Folgen von Gewalterfahrungen bei stationären und ambulanten Patientinnen und Patienten in psychologischer Behandlung/Beratung. Mit dem Studienansatz (anonyme Fragebogenuntersuchung, Interviews) werden auch die Akzeptanz und die Wirksamkeit einer psychologischen Unterstützung ermittelt.

Der Beitrag von Andreas Maercker und Tobias Hecker gibt einen Überblick über den internationalen Forschungsstand zu Gewalt- und Traumafolgen. Neben der Posttraumatischen Belastungsstörung (PTBS) kann es als Folge traumatischer Erfahrungen auch zur Entwicklung einer komplexen PTBS, einer anhaltenden Trauerstörung oder einer Anpassungsstörung kommen. Als indirekte Traumafolgestörungen gelten u. a. Depression, Substanzabhängigkeit und Persönlichkeitsstörungen. Als besonders erfolgreiche Therapieansätze beschreiben die Autoren verhaltenstherapeutische Interventionen, bei denen die Exposition gegenüber dem traumatischen Ereignis im Mittelpunkt steht.

Ingo Schäfer, Sven Barnow, Silke Pawils et al. befassen sich mit der Frage, inwieweit substanzbezogene Störungen als Ursache und/oder als Folge früher Ge- walt verstanden werden können. Der Beitrag basiert auf einem vom Bundesministerium für Bildung und Forschung (BMBF) geförderten Forschungsverbund „CANSAS“. Dieser zielt auf eine grundlagenwissenschaftliche Analyse der $\mathrm{Zu}$ sammenhänge zwischen "Substanzmissbrauch“ und „Gewalt", auf die Entwicklung und Bereitstellung von evidenzbasierten Behandlungsansätzen und auf die systematische Bewertung des Kindeswohls bei Kindern suchtkranker Eltern.

Der zweite Themenblock fokussiert Kinder als Opfer von Gewalt. Ute Ziegenhain, Anne Katrin Künster und Tanja Besier beschreiben Gewalt gegen Kinder und Jugendliche als ein weit verbreitetes Phänomen. Sie zeigen die Kontextabhängigkeit der festgestellten Prävalenzzahlen auf und dokumentieren, dass Gewalt gegen Kinder mit zahlreichen negativen Folgen für die körperliche, emotionale und psychosoziale Entwicklung des Kindes verbunden ist. Dargestellt werden weiterhin die zur Prävention von Gewalt inzwischen verfügbaren Strategien, wobei sie Programme, die auf die Förderung der elterlichen Erziehungs- und Beziehungskompetenzen zielen, als besonders vielversprechend einschätzen.

Silke Pawils und Franka Metzner knüpfen hier mit ihrem Literaturüberblick über Gewaltprävention im Kindesund Jugendalter direkt an. Im Beitrag werden die Forschungsbefunde zu Prävalenzen, typischen Entwicklungsverläufen und Prädiktoren von Gewaltverhalten im Kindesalter zusammengefasst und Entwicklungsverläufe und Folgen von Jugendgewalt gegenübergestellt. Weiterhin werden Programme zur Gewaltprävention für Kinder und Jugendliche vor dem Hintergrund verschiedener Settings, Zielgruppen und Zielvariablen vorgestellt.

Der Beitrag von Christiane Firnges und Stefanie Amann stellt die Evaluationsergebnisse eines schulbasierten Präventionstheaters im Rahmen der „Bundesweiten Initiative zur Prävention von sexuellem Missbrauch - Trau dich!“ vor. In Theaterepisoden werden Kindern Lösungen im Umgang mit Grenzverletzungen und Missbrauchssituationen aufgezeigt. Die Evaluation zeigt, dass das Theaterstück zu einem subjektiven Wissens- und Kompetenzzuwachs der Kinder beitragen konnte.

Wolfgang Melzer, Wilfried Schubarth, Lars Oertel et al. fokussieren das Thema "Gesundheitsbezogene Auswirkungen von Gewalt in der Schule“. Der Beitrag geht der Frage nach, wie sich Gewaltund Mobbingphänomene an deutschen Schulen entwickelt haben und welche Zusammenhänge es zur Gesundheit von Schülerinnen und Schülern gibt. Die empirische Basis bilden langjährige Untersuchungen der Autoren im Rahmen der internationalen „Health Behaviour in School-aged Children" (HBSC)-Studie.

Die Beiträge des dritten Themenblock betreffen Gewalt und Gewaltfolgen bei unterschiedlichen Gruppen von Erwachsenen. Karin Wieners und Marion Winterholler stellen bei ihrer Auseinandersetzung mit dem Thema „Häusliche und sexuelle Gewalt gegen Frauen" die 2013 veröffentlichten WHO-evidenzbasierten Leitlinien für den Umgang mit der Problematik in Versorgung, Aus, Fort- und Weiterbildung und Gesundheitspolitik in den Mittelpunkt. Die Autorinnen bestätigen die grundsätzliche Gültigkeit und Praktikabilität der Leitlinien für Deutschland, sehen hier aber bisher erhebliche Umsetzungsdefizite. Sie fordern deshalb die Entwicklung bundesweit geltender fachlicher Standards für die gesundheitliche Versorgung bei häuslicher und sexueller Gewalt, weiterhin einen (gesetzlichen) Auftrag für die Gesundheitsversorgung und die curriculare Verankerung des Themas in der Aus-, Fort- und Weiterbildung der Gesundheitsberufe.

Hildegard Lilly Graß, Britta Gahr und Stefanie Ritz-Timme befassen sich mit dem Umgang mit Opfern von häuslicher Gewalt in der ärztlichen Praxis. Der Beitrag stützt sich auf Ergebnisse eines vom Bundesministerium für Familie, Senioren, Frauen und Jugend (BMFSFJ) geförderten Modellprojekts zu diesem Thema („MIGG“-Projekt). Vorgestellt werden Ansätze für eine optimale Gewaltopferbetreuung im Praxisalltag. Ein wichtiges Thema stellen dabei Besonderheiten bei der Befundsicherung dar.

Albert Nienhaus, Claudia DrechselSchlund, Heike Schambortski und Anja 
Schablon behandeln das Thema "Gewalt und Diskriminierung am Arbeitsplatz“ und deren gesundheitliche Folgen. Sie beschreiben die sehr unterschiedlichen Formen von Gewalt am Arbeitsplatz und die vielfältigen Folgen für die Betroffenen und die Unternehmen. Zur Vermeidung von Gewalt am Arbeitsplatz schlagen die Autoren und Autorinnen unterschiedliche technische, organisatorische und persönliche Schutzmaßnahmen vor.

Tilman Steinert und Hans-Joachim Traub befassen sich mit dem Thema "Gewalt und psychisch Kranke" unter zwei Perspektive: nämlich Gewalt durch psychisch Kranke und Gewalt gegen psychisch Kranke. Ein erhöhtes Risiko für Gewaltdelikte bei Menschen mit einzelnen psychischen Erkrankungen gilt als gut gesichert. Gleichwohl sind die Rahmenbedingungen zu berücksichtigen. Die Autoren zeigen gleichzeitig auf, dass psychisch Kranke, insbesondere Frauen, gehäuft Opfer von Straftaten und Gewaltverbrechen sind.

Im abschließenden Beitrag weist Rolf D. Hirsch auf die erhebliche gesellschaftliche und gesundheitspolitische $\mathrm{Be}$ deutung des Themas „Gewalt gegen alte Menschen“ hin. Die bisherigen Ergebnisse zur Häufigkeit von Gewalt gegenüber alten Menschen sind sowohl im familiären wie stationären Bereich alarmierend hoch. Der Autor setzt sich mit Ursachen, der Psychodynamik und den Folgewirkungen von Gewalt gegenüber alten Menschen auseinander und diskutiert Ansatzpunkte zu deren Prävention.

Wir, die Herausgeber dieses Themenheftes, hoffen, dass es uns mit der Auswahl der Beiträge gelungen ist, Ihnen, liebe Leserinnen und Leser, die Vielschichtigkeit des Themas „Gewalt und Gesundheit" aufzuzeigen und Sie für die in einzelnen Bereichen bisher nicht öffentlich geführte Diskussion zu sensibilisieren.
Ihre

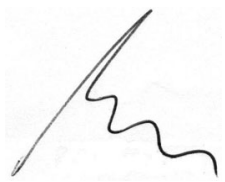

Uwe Koch-Gromus

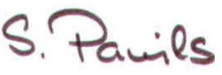

Silke Pawils

\section{Korrespondenzadresse}

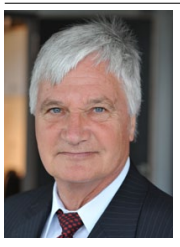

Prof. Dr. Dr. U. Koch-Gromus

Dekan der Medizinischen

Fakultät,

Universitätsklinikum

Hamburg-Eppendorf

Martinistraße 52, 20246

Hamburg

koch@uke.uni-hamburg.de

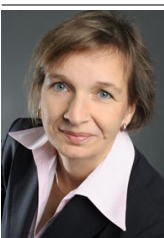

Dr. S. Pawils

Zentrum für Psychosoziale Medizin, Institut und Poliklinik für Medizinische Psychologie, Universitätsklinikum Hamburg-Eppendorf Martinistr. 52 (W26), 20246 Hamburg s.pawils@uke.de 